\title{
In-Situ Quantification of TEM Lamella Thickness and Ga Implantation in the FIB
}

\author{
Matthew Hiscock ${ }^{1}$, Michael Dawson ${ }^{2}$, Christian Lang ${ }^{1}$, Cheryl Hartfield ${ }^{2}$ and Peter Statham ${ }^{1}$ \\ 1. Oxford Instruments NanoAnalysis, Halifax Road, High Wycombe, HP12 3SE, UK \\ 2. Oxford Instruments NanoAnalysis, 10410 Miller Rd., Dallas, TX 75238
}

Focused Ion beam (FIB) based tools have become the preferred method to prepare TEM lamellas, largely due to their high resolution imaging capabilities used to identify the site of interest. The quality and thickness of samples has become paramount in order to take full advantage of the ever increasing resolution in aberration corrected TEMs and accurately controlling the lamella thickness at the same time as minimising any amorphisation caused by ion implantation is challenging. For instruments combining a focused ion beam with an electron beam methods based on either back scattered electron contrast [1] or transmissivity of electrons [2] have been demonstrated. However, these methods only work on homogenous samples without compositional variations and require for the contrast to be calibrated using the same material. They also don't provide any information on ion implantation or surface amorphisation and can greatly affect the quality of the TEM image obtainable from the lamella.

Here we show a method that uses X-rays generated by the electron beam - lamella interaction to accurately and rapidly measure the lamella composition and thickness. A similar method has been used to measure a wide range of thin films and layers on bulk substrates [3]. In order to measure the thickness and composition of the lamella, we used Oxford Instruments' AZtec LayerProbe software and X-Max 150 EDS detectors to acquire and process EDS spectra. The LayerProbe software refines a starting model of the sample structure against the EDS spectra to calculate the film thickness and composition of the layers. The starting model comprises the layer sequence in the sample and a substrate material. As the TEM lamella is a free-standing layer the substrate is defined as comprising an element that is not contained in the lamella and only weakly scatters electrons such as Beryllium. The first layer is defined as the material comprising the lamella and its thickness is used as a measure for the lamella thickness. The top layer can be defined to contain the element used as the ion source (e.g. Gallium) to obtain a measure of the degree of ion implantation in the specimen.

Fig. 1a shows an electron image of a TEM lamella prepared from a Ni based superalloy (alloy 600). A grid of spectra acquired from the lamella was used to calculate the composition and thickness at every point. Fig. 1b shows a surface plot of the lamella thickness and Fig. 1c the Ga thickness. The thickness of the lamella is clearly decreasing from the area close to the weld towards the free end of the lamella with the lowest thickness of the lamella measured at around $75 \mathrm{~nm}$. The Ga thickness profile shows a different trend with an increase Ga thickness close to the left lower corner and also close to the weld. The thickness of the Ga layer over the thinnest area of the specimen is considerably below $5 \mathrm{~nm}$ which indicates that most of the Ga implanted during the high energy milling has been removed subsequently. Fig. 2 shows an X-ray map of a TEM lamella prepared from a silicon semiconductor device. The device structures containing $\mathrm{Cu}$ and $\mathrm{W}$ are clearly visible in the X-ray maps. One of the $\mathrm{Cu}$ lines fades and disappears from the right side to the left of the lamella. This indicates that the line runs at an angle to the direction of the FIB cut. With LayerProbe it is possible to measure the projected $\mathrm{Cu}$ thickness and $\mathrm{Si}$ thickness from X-ray spectra reconstructed from the X-ray map. By comparing measurements taken from the right side of the lamella with measurements towards the left side we can see how the thickness increase of the lamella affects the ratio of device vs surrounding Si matrix for both the $\mathrm{W}$ and $\mathrm{Cu}$ rich device areas. 
Most FIB-SEMs are already equipped with an EDS detector therefore no additional hardware is necessary to implement this new technique. This enables its rapid adaptation as a standard metrology tool to support the preparation of high quality TEM lamellas.

References:

[1] A. R. Hall, Microscopy and Microanalysis 19 (2013), p. 740.

[2] U. Golla-Schindler, Conference Proceedings EMC (2008), p 667.

[3] C. Lang et al., Microscopy and Microanalysis 19 (2013), p. 1872.

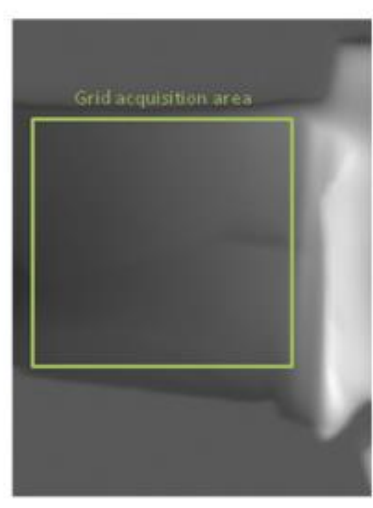

(a)

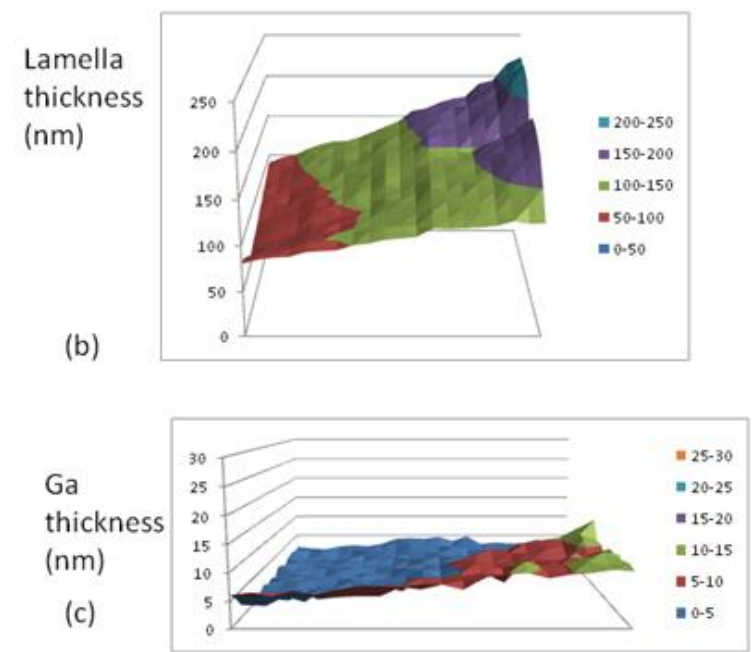

Figure 1. (a) shows an electron image of a TEM lamella of Ni superalloy 600 and the area for which the lamella thickness in (b) and the equivalent Ga thickness (c) have been calculated.

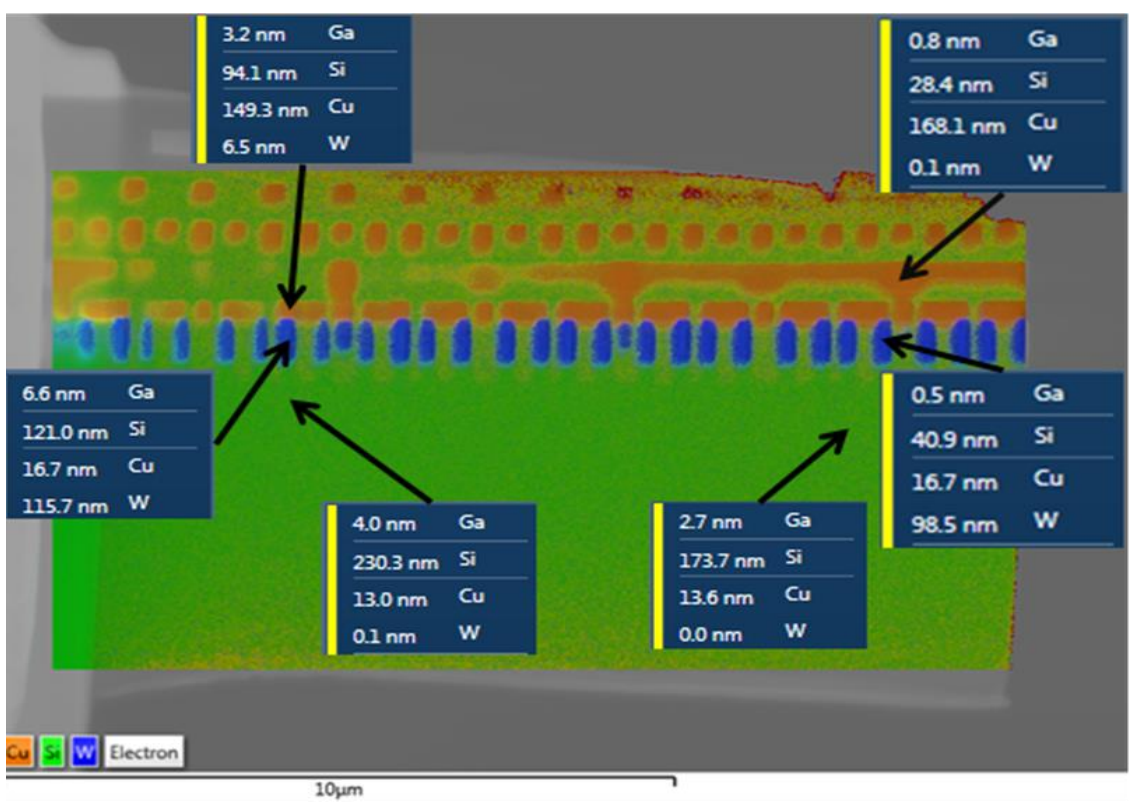

Figure 2. The local lamella thickness as well as the contribution of different device layers to this thickness was calculated from spectra reconstructed from an X-ray map. 